Pak. j. sci. ind. res. Ser. B: biol. sci. 2021 64B(1) 64-74

\title{
Effect of Salinity on Emergence and Early Growth Stages of Aromatic and Non-Aromatic Rice (Oryza sativa L.) Genotypes
}

\author{
Ghulam Sarwar Channa ${ }^{a}$, Abdul Razak Mahara, Inayatullah Rajparb, \\ Abdul Hafeez Memon ${ }^{c}$, Muneer Ali Bhagat ${ }^{a}$, Mumtaz Ali Saand ${ }^{d}$, \\ Ameer Ahmed Mirbahar ${ }^{b}$ and Muzafar Sirohib* \\ a'Department of Botany, Shah Abdul Latif University, Khairpur, Pakistan \\ ${ }^{b}$ Department of Soil Science, Sindh Agriculture University, Tandojam, Pakistan \\ ${ }^{\mathrm{c}}$ Agriculture Research Institute, Tandojam, Pakistan \\ ${ }^{\mathrm{d}}$ Centre for Biodiversity and Conservation, Shah Abdul Latif University Khairpur, Pakistan
}

(received July 31, 2018; revised January 13, 2019; accepted January 17, 2019)

\begin{abstract}
Salinity hampers the production of many field crops in the region including rice (Oryza sativa L.), while commonly classified as salt sensitive within the same species, the level of sensitivity varies between genotypes. This study investigated the salinity tolerance of 28 rice genotypes, including 9 aromatics and 19 non-aromatics. Sixty seeds of each genotype were initially sown in trays $(24 \times 18$ inches $)$ during the $1^{\text {st }}$ week of June, by of using four salt treatments $\left(0,40,80\right.$ and $\left.120 \mathrm{mM} \mathrm{NaCl}+\mathrm{CaCl}_{2} @ 20: 1\right)$. The experiment was laid down in a completely randomized design with four replicates in laboratory conditions, at Shah Abdul Latif University Khairpur, Sindh, for the period of twenty-five days. A significant reduction in agro-morphological parameters was observed against all salinity levels. Based on reduction in dry matter yield, all rice genotypes were found tolerant at $40 \mathrm{mM}$. Eleven rice genotypes were found tolerant, fourteen were moderately tolerant, one was moderately sensitive and remaining two genotypes were found sensitive at $80 \mathrm{mM}$ salinity level. Furthermore, none of the genotypes were able to with stand $120 \mathrm{mM}$ of salinity. The genotypes Khushboo, DR-83 and Mahek performed meager and showed more than $50 \%$ reduction over control and categorised as sensitive, with the genotypes Latifee, DR-67 and DR-92, DR-51 and IR-6 are categorised as tolerant with a reduction of less than $20 \%$ over control based on dry matter yield reduction against all salinity treatments at the early seedling stage. However, these genotypes cannot be justified as tolerant only on the basis of their improved performance at early growth stage. Hence, these genotypes are suggested to be studied further at other advanced growth stages up to maturity to evaluate their response under a saline environment.
\end{abstract}

Keywords: salinity tolerance, aromatic and non-aromatic rice, genotypes, germination, survival percentage, dry matter yield

\section{Introduction}

Rice, a major export commodity, for export, play a key role in Pakistan agrarian based economy. The country earned 26.5 million US\$ during 2017 through its export (PBS, 2018). It is the third largest crop in terms of area, cultivating over 9,050,000 hectares and second principal food grain crop of Pakistan, produced 26,500,000 tonnes during 2017-18. Punjab and Sindh are the major rice producing provinces of Pakistan, contributing approximately $90 \%$ of the overall rice production (PBS, 2018). Rice is one of the nutritionally imperious grain crops feeding more than three billion people around the world (Ghosh et al., 2016; Mohammadi-Nejad et al., 2010). A $100 \mathrm{~g}$ of rice provide $345 \mathrm{Kcal}$ energy, $78.2 \mathrm{~g}$ of carbohydrates, $6.8 \mathrm{~g}$ of protein and a significant amount

*Author for correspondence;

E-mail: muzafar.sirohi@salu.edu.pk of suggested Zinc and Niacin (Ali et al., 2014; Gopalan et al., 2007). Rice varieties with some special traits such as fragrance, better taste and higher cooking quality also provide extra value in socio-economic aspects.

Salinity is one of the ecological challenges after drought, reducing crop production over 800 million hectares throughout the world (Islam et al., 2013). Generally, the $\mathrm{Na}^{+}$and $\mathrm{Cl}^{-}$ions resulting from $\mathrm{NaCl}$, are the major cause of salinity. A high concentration of such ions may affect the functioning of plant cell, resulting in reduced growth and lessen yield (Läuchli and Grattan, 2007). High salinity in the flooded soil is one of the main factors that depress water availability to root cells of rice plants due to reduced osmotic potential, consequently, inhibiting growth, development and grain yield of rice (Ganie et al., 2016; Molla et al., 2015). Salinity causes oxidative stress and disparity in nutrition, due 
to high concentration of specific ions unbalancing osmotic regulation (Ashraf, 2010; Noreen et al., 2010). This further inhabits germination, affects the growth of seedlings, and crush leaf enlargement, subsequently providing small leaf blade for photosynthetic activities and less dry matter of plant (Ahmad et al., 2010; Ashraf, 2010).

During the early seedling (2-3 leaf) stage, rice remains very sensitive studied by (Mondal and Borromeo, 2016). Salinity reduces seedling survival and results in low crop standing (Pushpam and Rangasamy, 2000). Salinity reduces the weight of the shoot, the number of leaves, shoot and root length and the surface area of the roots (Meloni et al., 2001). Some high concentrations of $\mathrm{Na}^{+}$ and/or $\mathrm{Cl}^{-}$in plants cause wilting of leaf tips and necrosis (Zafar et al., 2015). Salinity stressed suppress development can be distinguished by measuring effects immediately upon salt exposure or after several days to weeks (Roy et al., 2014). Soil salinity is one of many factors that poses a major challenge to sustainable agriculture in Pakistan (Hussain et al., 2012). Most of the saline soils have originated from various natural processes, such as rocks weathering movement of salt traces through irrigation water (Munns and Tester, 2008).

In Asia, salinity has affected more than twenty million hectares of arable land and loss about half of the predictable fertile land (Huyen et al., 2013; Nazar et al., 2011). Moreover, the human population around the globe is increasing rapidly. Either cropping area or production is to be increased to feed this growing population of the world. The supply of agricultural land availability is slowly declining with speed as population grows, as much of the land is being converted into residential and commercial areas for community. On the other hand, agriculture is suffering from serious damage of biotic and abiotic stresses. Scientists around the globe are putting their best efforts to improve genotypes to combat stress affected environments and meet the challenges of the present era (Sankar et al., 2011). The screening of salt tolerant species is a reliable method to cope the salinity and produce a better production (Shannon et al., 1998). Therefore, this study was conducted to screen out the salt-tolerant rice genotypes at early seedling stages and to test their salt tolerance levels in saline environment.

\section{Materials and Methods}

In the $1^{\text {st }}$ week of June 2016, the experiment was conducted at Department of Botany, Shah Abdul University
Khairpur, Sindh, Pakistan. Twenty eight rice genotypes were selected, including 09 aromatic (Mahek, Khushboo, DR-61, DR-62, DR-63, DR-66, DR-67, Super Basmati and Lateefi), and 19 non-aromatic (Kanwal-95, Shahkar, Sarshar, Sada Hayat, Shadab, Shandar, NIA-19-A, NIA625, DR-50, DR-51, DR-52, DR-57, DR-59, DR-82, DR-83, DR-92, IR-6, IR-8 and Shua-92 was used as salt-tolerant check variety) for analysis. The seeds of genotypes were sourced from RRI Dokri, Sindh, Pakistan and NIA Tandojam, Sindh, Pakistan. The seeds were sterilized for $30 \mathrm{~min}$ on the surface with $1 \%$ industrial bleach and washed four times with distilled water. After rinsing, sixty seeds of each genotype were sown in $10 \mathrm{Kg}$ air dried soil filled trays $(24 \times 18$ inches $)$. The soil was analyzed before experiments (Table 1 ). The trays were arranged in completely randomized design (CRD) with four repeats and four salt treatments (0, 40, 80 and $\left.120 \mathrm{mM}, \mathrm{NaCl}+\mathrm{CaCl}_{2} @ 20: 1\right)$ were applied. The salt solution was prepared by following method. For the conversion of molar into $\mathrm{mM}$ following formula was used:

$$
\mathrm{mM}=\frac{\text { Molar weight of } \mathrm{NaCl}}{1000} \times \text { salt treatments }
$$

The resulting weight of salt is added in distilled water per liter to make a salt solution. The temperature of the laboratory was maintained at $28^{\circ} \mathrm{C}$.

Emergence percentage was measured at interval of one week of sowing. Twenty five days old seedlings were harvested and washed with distilled water. Survival percentage, shoot height and root length $(\mathrm{cm})$, shoot and root dry weight $(\mathrm{g})$, shoot/root ratio and dry matter yield $(\mathrm{g})$ were recorded at the time of seedlings harvest. Seedlings were wrapped in paper bag and kept in an

Table 1. Physico-chemical properties of soil used in experiment

\begin{tabular}{ll}
\hline \hline Soil properties & Value \\
\hline Texture & \\
$\quad$ Sand & $25.2 \%$ \\
$\quad$ Silt & $41.3 \%$ \\
$\quad$ Clay & $33.5 \%$ \\
Textural class & Clay loam \\
Electrical conductivity (1:5) & $2.2 \mathrm{~d} / \mathrm{Sm}$ \\
pH (1:5) & 7.4 \\
Organic matter & $0.75 \%$ \\
$\mathrm{CaCO}_{3}$ & $6.0 \%$ \\
\hline \hline
\end{tabular}


oven at $65{ }^{\circ} \mathrm{C}$ for $24 \mathrm{~h}$ to a constant dry weight. The germination percentage (GP) and survival percentage (SP) was calculated using the formula suggested by Raun et al. (2002). Whereas, percent reduction over control (PROC) was computed using the formula suggested by Ali et al. (2014), as following:

$$
\begin{aligned}
& \mathrm{GP}=\frac{\text { Total number of germinated seeds }}{\text { Total number of germinated seedling }} \times 100 \\
& \mathrm{SP}=\frac{\text { Total number of survival seedling }}{\text { Total number of germinated seedling }} \times 100 \\
& \text { Volume in control - Value in saline } \\
& \text { PROC }=\frac{\text { environment }}{\text { Value in control }} \times 100
\end{aligned}
$$

Genotypes were categorised as tolerant $(\mathrm{T})$ having 0 $20 \%$ reduction, moderately tolerant (MT) and moderately sensitive (MS), having $21-40 \%$ and $41-60 \%$ reduction respectively while, sensitive (S) more than $60 \%$ reduction of their aggregate dry material (shoot and root) at various levels of salinity stress (Ologundudu et al., 2014; Hakim et al., 2010). The data were analyzed by performing two-way ANOVA $(\mathrm{P}<0.05)$ and means were compared by least significant difference (LSD) using statistical software namely "Statistix version 8.1".

\section{Results and Discussion}

Germination percentage (GP). The germination of all aromatic and non-aromatic rice genotypes significantly decreased when exposed to salinity treatments (Table 2). A negligible effect of salinity was observed at $40 \mathrm{mM}$ salinity with least (14.6) average PROC of all genotypes (Fig.1a). However, genotype DR-63 showed maximum (37.77) PROC even at this level of salinity. The most severe effect of salinity was observed at $120 \mathrm{mM}$ salt concentration, where maximum (47.86) average PROC of all genotypes was recorded. The genotypes DR-92, DR-51 and Latifee performed better, both showed least $(\leq 16.51)$ average PROC at all salinity levels, whereas genotypes DR-63, DR-61 and Sada Hayat germinated poorly in saline environment and showed maximum $(>50)$ average PROC as compared to other genotypes. Generally, PROC increases with the rise in salinity level (Fig.1a).

Survival percentage (SP). Increased salinity levels significantly decreased the survival percentage of all rice genotypes (Table 2) as compared to control salinity treatment. Salinity level $120 \mathrm{mM}$ showed most harmful effects on seedling survival, where more than $60 \%$ reduction was observed as compared to control (Fig. 1b). Genotypes DR-83 and Khushboo could not with stand the hazardous effect of salinity and completely died at $80 \mathrm{mM}$ salinity. Genotypes DR-92, DR-51 and IR-6 remained successful with least average $(\leq 21)$ PROC.

Shoot height and root length. Shoot height of all aromatic and non-aromatic rice genotypes was reduced when exposed to salt stress (Table 3). Mild effect of salinity was observed at $40 \mathrm{mM}$ salinity, where on an average less than ten PROC was recorded. The most significant effect of salinity was noted at $120 \mathrm{mM}$ salinity treatment, where on an average 56.62 PROC was observed. The maximum $(68.98,68.73$ and 41.67$)$ average PROC was observed in genotypes Khusboo, DR-83 and DR-57 respectively, whereas minimum average $(<20)$ PROC was recoded in genotypes Latifee, DR-92 and DR-51 (Fig.1c). Similarly, root length was also decreased with increased levels of salinity (Table 4). The minimum (11.24) average PROC in root length was observed at $40 \mathrm{mM}$ salinity which raised to maximum (61.91) average PROC at maximum (120 $\mathrm{mM})$ salinity level. The genotypes DR-92, NIA-19A and Super Basmati showed minimum $(\leq 26)$ average PROC in root length at all salinity levels, whereas maximum (69.07, 68.49 and 50.37) average PROC for same trait at all salinity treatments was recorded in genotypes Khushboo, DR-83 and DR-52, respectively (Fig.1d).

Shoot and root dry weight. Shoot dry weight of all rice genotypes was significantly reduced with higher salinity levels as compared to control (Table 3 ). All genotypes performed well with a least PROC $(<9 \%)$ at $40 \mathrm{mM}$ salinity treatment (Fig.1e). DR-92, Latifee and DR-51 showed better response with minimum (18.82, 22.64 and 22.75) average PROC, whereas Khushboo, DR-83 and Mahek performed meager and showed maximum average PROC at all salinity levels (Fig.1e). Similarly, root dry weight of all genotypes was also decreased with increased salinity levels (Table 4), on average, at 40,80 and $120 \mathrm{mM}$ salinity, root dry weight of all genotypes reduced (8.24, 24.54 and $51.48 \%$ ) respectively as compared to control.

The lowest $(14.18,16.99$ and $17.39 \%)$ reduction on average at all salinity levels were observed in genotypes DR-67, DR-92 and DR-51 respectively, whereas highest $(68.69,67.50$ and $50.45 \%)$ average reduction at all 
salinity levels in root dry weight were recorded in genotypes Khushboo, DR-83 and Mahek respectively as compared to control (Fig.1f).

Shoot/root ratio. In all genotypes, shoot/root ratio was significantly decreased with increased salinity levels (Table 5). The highest (2.51, 2.44 and 2.42) shoot/root ratio at all salinity levels were observed in genotypes DR-92, DR-51 and Latifee respectively, whereas lowest $(0.94,1.27$ and 1.28$)$ shoot/root ratios were recorded in genotypes DR-83, IR-8 and DR-52, respectively. The more average PROC ( $>60)$ was observed in genotypes Khushboo, DR-83 and Shandar respectively, while mild average PROC $(\leq 13.5)$ was recorded in genotypes DR-92, DR-51 and IR-6 (Fig.1g).

Classification of genotypes. All genotypes were classified as tolerant (T), medium tolerant (MT), medium sensitive (MS), and sensitive (S) on the basis of dry matter production at different salinity levels (Table 5). All rice genotypes fall in the category of T at $40 \mathrm{mM}$. Eleven rice genotypes fall in the category of $\mathrm{T}$, fourteen in the category of MT, one in the category of MS and remaining two genotypes fall in the category of $\mathrm{S}$ at 80 $\mathrm{mM}$ salinity level. No any rice genotype could qualify for T category at $120 \mathrm{mM}$ salinity.

Table 2. Effect of salinity on germination and survival percentages of aromatic and non-aromatic rice genotypes grown in solution culture. The number shows the mean germination and survival under various salinity treatments. $\left(\mathrm{NaCl}+\mathrm{CaCl}_{2} @ 20: 1\right.$ salt concentrations (mM))

\begin{tabular}{|c|c|c|c|c|c|c|c|c|c|c|}
\hline \multirow[t]{2}{*}{ Genotypes } & \multicolumn{5}{|c|}{ Germination percentage (GP) } & \multicolumn{5}{|c|}{ Survival percentage (SP) } \\
\hline & $\mathrm{T} 1$ & $\mathrm{~T} 2$ & T3 & T4 & $\begin{array}{l}\text { Genotype } \\
\text { mean }\end{array}$ & $\mathrm{T} 1$ & $\mathrm{~T} 2$ & T3 & $\mathrm{T} 4$ & $\begin{array}{l}\text { Genotype } \\
\text { mean }\end{array}$ \\
\hline Sarshar & 85.0 & 75.4 & 70.5 & 53.0 & 71.0 & 94.1 & 93.0 & 76.9 & 34.3 & 74.6 \\
\hline Shadab & 82.3 & 75.0 & 55.0 & 30.0 & 60.6 & 94.0 & 84.4 & 69.0 & 34.4 & 70.5 \\
\hline Shandar & 86.6 & 78.4 & 71.3 & 48.0 & 71.1 & 89.8 & 85.7 & 51.1 & 34.1 & 65.2 \\
\hline Shua-92 & 87.2 & 81.4 & 75.2 & 70.0 & 78.5 & 96.3 & 93.2 & 83.2 & 67.5 & 85.0 \\
\hline NIA-19A & 86.0 & 76.6 & 70.1 & 65.0 & 74.4 & 94.4 & 89.1 & 73.1 & 54.0 & 77.7 \\
\hline NIA-625 & 86.6 & 70.0 & 50.4 & 30.0 & 59.3 & 85.0 & 71.3 & 56.6 & 27.5 & 60.1 \\
\hline DR-50 & 85.0 & 70.5 & 50.3 & 35.0 & 60.2 & 95.3 & 76.1 & 72.9 & 20.6 & 66.2 \\
\hline DR-57 & 86.0 & 73.4 & 70.0 & 46.1 & 68.9 & 88.4 & 73.5 & 55.9 & 0.0 & 54.4 \\
\hline DR-83 & 85.0 & 68.3 & 40.0 & 30.0 & 55.8 & 90.8 & 76.4 & 0.0 & 0.0 & 41.8 \\
\hline DR-52 & 86.0 & 75.0 & 70.1 & 44.8 & 69.0 & 88.0 & 80.0 & 69.4 & 0.0 & 59.3 \\
\hline DR-51 & 87.0 & 83.5 & 76.4 & 60.3 & 76.8 & 94.1 & 91.2 & 74.3 & 57.6 & 79.3 \\
\hline DR-82 & 85.0 & 66.2 & 45.0 & 30.1 & 56.6 & 84.7 & 76.6 & 64.2 & 30.3 & 63.9 \\
\hline DR-92 & 88.1 & 81.6 & 73.0 & 71.1 & 78.4 & 94.4 & 91.4 & 68.4 & 66.7 & 80.2 \\
\hline IR-6 & 87.0 & 76.1 & 73.3 & 68.0 & 76.1 & 94.3 & 90.5 & 82.1 & 50.0 & 79.2 \\
\hline IR-8 & 85.0 & 76.0 & 50.5 & 30.0 & 60.4 & 88.6 & 71.2 & 65.6 & 28.5 & 63.5 \\
\hline Sada Hayat & 85.0 & 61.6 & 38.0 & 24.8 & 52.4 & 86.3 & 75.0 & 62.9 & 29.0 & 63.3 \\
\hline Kanwal-95 & 85.0 & 70.0 & 39.9 & 34.6 & 57.4 & 90.6 & 75.0 & 66.1 & 29.0 & 65.2 \\
\hline Shahkar & 86.4 & 73.1 & 70.0 & 64.6 & 73.5 & 94.0 & 80.8 & 76.1 & 52.2 & 75.8 \\
\hline DR-59 & 82.0 & 70.4 & 59.6 & 45.0 & 64.2 & 88.2 & 75.0 & 62.2 & 35.0 & 65.1 \\
\hline Mahek & 78.0 & 71.6 & 68.3 & 24.8 & 60.7 & 87.0 & 77.0 & 64.9 & 0.0 & 57.2 \\
\hline Khushboo & 81.2 & 65.0 & 45.0 & 25.0 & 54.1 & 87.0 & 77.1 & 0.0 & 0.0 & 41.0 \\
\hline DR-62 & 84.0 & 74.5 & 60.1 & 51.3 & 67.5 & 87.0 & 78.2 & 67.6 & 30.0 & 65.7 \\
\hline DR-66 & 94.0 & 85.1 & 81.5 & 50.0 & 77.7 & 94.0 & 85.1 & 81.5 & 50.0 & 77.7 \\
\hline DR-67 & 85.0 & 76.8 & 74.6 & 60.1 & 74.1 & 94.0 & 79.2 & 76.1 & 62.0 & 77.8 \\
\hline DR-63 & 80.4 & 50.0 & 35.1 & 28.0 & 48.4 & 95.3 & 72.7 & 52.2 & 28.0 & 62.1 \\
\hline Super Basmati & 86.0 & 81.0 & 70.0 & 36.5 & 68.4 & 94.3 & 88.2 & 74.3 & 52.6 & 77.4 \\
\hline DR-61 & 76.2 & 50.0 & 35.3 & 25.2 & 46.7 & 94.5 & 72.7 & 54.8 & 37.4 & 64.8 \\
\hline Latifee & 85.0 & 76.2 & 71.7 & 65.0 & 74.5 & 94.2 & 88.0 & 73.0 & 60.0 & 78.8 \\
\hline \multirow[t]{2}{*}{ Treatment mean } & 84.85 & 72.59 & 60. & 44.5 & & 91.4 & 81.0 & 63.4 & 34.7 & \\
\hline & \multicolumn{2}{|c|}{ Genotype (G) } & Salinity & GXS & & \multicolumn{2}{|c|}{ Genotype (G) } & Salinity & GX S & \\
\hline S.E.D & \multicolumn{2}{|l|}{0.62} & 0.23 & 1.25 & & \multicolumn{2}{|l|}{0.48} & 0.18 & 0.96 & \\
\hline L.S.D (0.05\%) & \multicolumn{2}{|c|}{$1.23 * * *$} & $0.46^{* * *}$ & $2.47 * * *$ & & \multicolumn{2}{|c|}{$0.94 * * *$} & 0.35 & $1.89 * * *$ & \\
\hline
\end{tabular}


Although eight genotypes were found successful for MT, eleven for MS and remaining nine were categorised as $\mathrm{S}$ at $120 \mathrm{mM}$ salinity.

In Pakistan, agriculture is facing plenty of problems and issues regarding crop production (Muzaffar et al., 2015; Rao et al., 2013). Several approaches are being attempted to cope against those problems and issues (Awan et al., 2015; Nasir et al., 2014). Soil salinity, an abiotic stress is also one of the major problems which not only reduce the crop growing area but also its yield throughout the world (Kronzucker et al., 2008). Rice is susceptible to salt-stress, especially during the period of seedling growth (Zafar et al., 2015). It is therefore, vital to screen out the best genotypes that produce better yield in saline conditions (Zeng and Shannon, 2002).

In present study, the influence of salinity remained adverse on emergence, survival and early growth of aromatic and non-aromatic rice genotypes. Results showed a significant reduction in almost all agronomic parameters. However, the rate of reduction varied in both aromatic and non-aromatic rice genotypes. The symptoms of salt injury could be visualized physically.

Table 3. Effect of salinity on shoot height and shoot dry weight of aromatic and non-aromatic rice genotypes grown in solution culture. The number shows the mean shoot height $(\mathrm{cm})$ and shoot dry weight $(\mathrm{g})$ under various salinity treatments. $\left(\mathrm{NaCl}+\mathrm{CaCl}_{2} @ 20: 1\right.$ salt concentrations $\left.(\mathrm{mM})\right)$

\begin{tabular}{|c|c|c|c|c|c|c|c|c|c|c|}
\hline \multirow[t]{2}{*}{ Genotypes } & \multicolumn{5}{|c|}{ Shoot height $(\mathrm{cm})$} & \multicolumn{5}{|c|}{ Shoot dry weight (g) } \\
\hline & T1 & $\mathrm{T} 2$ & T3 & $\mathrm{T} 4$ & $\begin{array}{l}\text { Genotype } \\
\text { mean }\end{array}$ & $\mathrm{T} 1$ & $\mathrm{~T} 2$ & T3 & $\mathrm{T} 4$ & $\begin{array}{l}\text { Genotype } \\
\text { mean }\end{array}$ \\
\hline Sarshar & 15.1 & 13.4 & 11.9 & 5.9 & 11.6 & 0.089 & 0.08 & 0.066 & 0.027 & 0.066 \\
\hline Shadab & 16.0 & 12.6 & 11.1 & 7.9 & 11.9 & 0.097 & 0.082 & 0.068 & 0.023 & 0.068 \\
\hline Shandar & 15.5 & 12.6 & 11.4 & 9.0 & 12.1 & 0.088 & 0.076 & 0.067 & 0.033 & 0.066 \\
\hline Shua-92 & 16.2 & 14.8 & 13.5 & 10.2 & 13.7 & 0.131 & 0.123 & 0.111 & 0.061 & 0.107 \\
\hline NIA-19A & 16.7 & 15.4 & 12.5 & 10.7 & 13.8 & 0.089 & 0.081 & 0.074 & 0.049 & 0.073 \\
\hline NIA-625 & 15.7 & 13.7 & 12.9 & 8.4 & 12.7 & 0.084 & 0.078 & 0.06 & 0.027 & 0.062 \\
\hline DR-50 & 15.7 & 14.5 & 12.7 & 7.0 & 12.5 & 0.076 & 0.072 & 0.058 & 0.028 & 0.059 \\
\hline DR-57 & 14.9 & 13.9 & 12.2 & 0.0 & 10.3 & 0.081 & 0.077 & 0.063 & 0 & 0.055 \\
\hline DR-83 & 13.2 & 12.4 & 0.0 & 0.0 & 6.4 & 0.074 & 0.071 & 0 & 0 & 0.036 \\
\hline DR-52 & 14.9 & 13.7 & 12.4 & 0.0 & 10.3 & 0.083 & 0.073 & 0.058 & 0 & 0.054 \\
\hline DR-51 & 15.7 & 14.8 & 12.7 & 10.5 & 13.4 & 0.126 & 0.115 & 0.102 & 0.075 & 0.105 \\
\hline DR-82 & 16.2 & 15.0 & 12.4 & 9.6 & 13.3 & 0.081 & 0.07 & 0.06 & 0.045 & 0.064 \\
\hline DR-92 & 16.8 & 15.6 & 13.8 & 12.1 & 14.6 & 0.124 & 0.121 & 0.102 & 0.079 & 0.107 \\
\hline IR-6 & 16.1 & 15.5 & 12.7 & 10.4 & 13.7 & 0.116 & 0.106 & 0.081 & 0.07 & 0.093 \\
\hline IR-8 & 17.8 & 14.7 & 13.1 & 7.1 & 13.2 & 0.077 & 0.068 & 0.06 & 0.035 & 0.06 \\
\hline Sada Hayat & 16.2 & 14.5 & 12.4 & 7.9 & 12.8 & 0.082 & 0.078 & 0.06 & 0.041 & 0.065 \\
\hline Kanwal-95 & 19.0 & 15.2 & 14.8 & 9.9 & 14.7 & 0.077 & 0.065 & 0.058 & 0.045 & 0.061 \\
\hline Shahkar & 15.8 & 14.7 & 14.1 & 8.7 & 13.3 & 0.117 & 0.109 & 0.081 & 0.07 & 0.094 \\
\hline DR-59 & 14.6 & 13.3 & 12.3 & 7.6 & 11.9 & 0.076 & 0.07 & 0.057 & 0.037 & 0.06 \\
\hline Mahek & 14.7 & 14.0 & 12.5 & 0.0 & 10.3 & 0.106 & 0.099 & 0.047 & 0 & 0.063 \\
\hline Khushboo & 14.4 & 13.4 & 0.0 & 0.0 & 7.0 & 0.101 & 0.091 & 0 & 0 & 0.048 \\
\hline DR-62 & 17.1 & 16.3 & 13.9 & 5.4 & 13.2 & 0.084 & 0.076 & 0.062 & 0.03 & 0.063 \\
\hline DR-66 & 17.7 & 16.3 & 14.3 & 6.4 & 13.7 & 0.081 & 0.072 & 0.066 & 0.038 & 0.064 \\
\hline DR-67 & 23.1 & 20.5 & 16.0 & 13.5 & 18.3 & 0.108 & 0.095 & 0.08 & 0.07 & 0.088 \\
\hline DR-63 & 17.2 & 13.2 & 12.3 & 8.3 & 12.8 & 0.079 & 0.074 & 0.062 & 0.025 & 0.06 \\
\hline Super Basmati & 18.1 & 16.5 & 12.9 & 9.5 & 14.3 & 0.096 & 0.086 & 0.075 & 0.055 & 0.078 \\
\hline DR-61 & 16.5 & 15.1 & 12.5 & 6.4 & 12.6 & 0.081 & 0.078 & 0.06 & 0.038 & 0.064 \\
\hline Latifee & 15.7 & 14.9 & 14.0 & 10.7 & 13.8 & 0.106 & 0.102 & 0.085 & 0.059 & 0.088 \\
\hline \multirow[t]{2}{*}{ Treatment mean } & 16.3 & 14.7 & 12.04 & 7.24 & & 0.093 & 0.085 & 0.065 & 0.038 & \\
\hline & & \multicolumn{2}{|c|}{ Genotype (G) } & Salinity & GXS & & \multicolumn{2}{|c|}{ Genotype (G) } & \multicolumn{2}{|c|}{ Salinity (S) G X S } \\
\hline \multicolumn{2}{|l|}{ S.E.D } & \multicolumn{2}{|l|}{0.42} & 0.15 & 0.84 & & \multicolumn{2}{|l|}{1.90} & 7.20 & 3.81 \\
\hline \multicolumn{2}{|c|}{ L.S.D (0.05\%) } & \multicolumn{2}{|c|}{$0.82 * * *$} & $0.31 * * *$ & $0.65 * * *$ & & \multicolumn{2}{|c|}{$3.75 * * *$} & $14.42 * * *$ & $7.51 * * *$ \\
\hline
\end{tabular}


Burning of older leaf tips were noticed at initial stage which led to completely dying of leaf. Germination of all rice genotypes including aromatics and non-aromatics were affected by salinity. Reduction in their germination

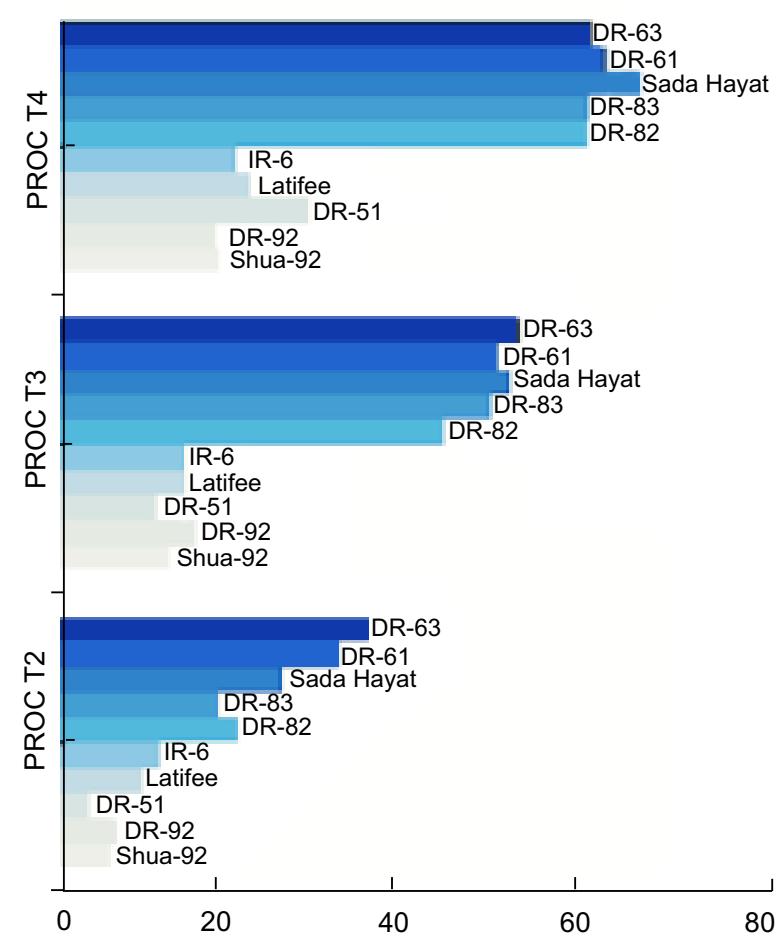

(a) Germination percentage

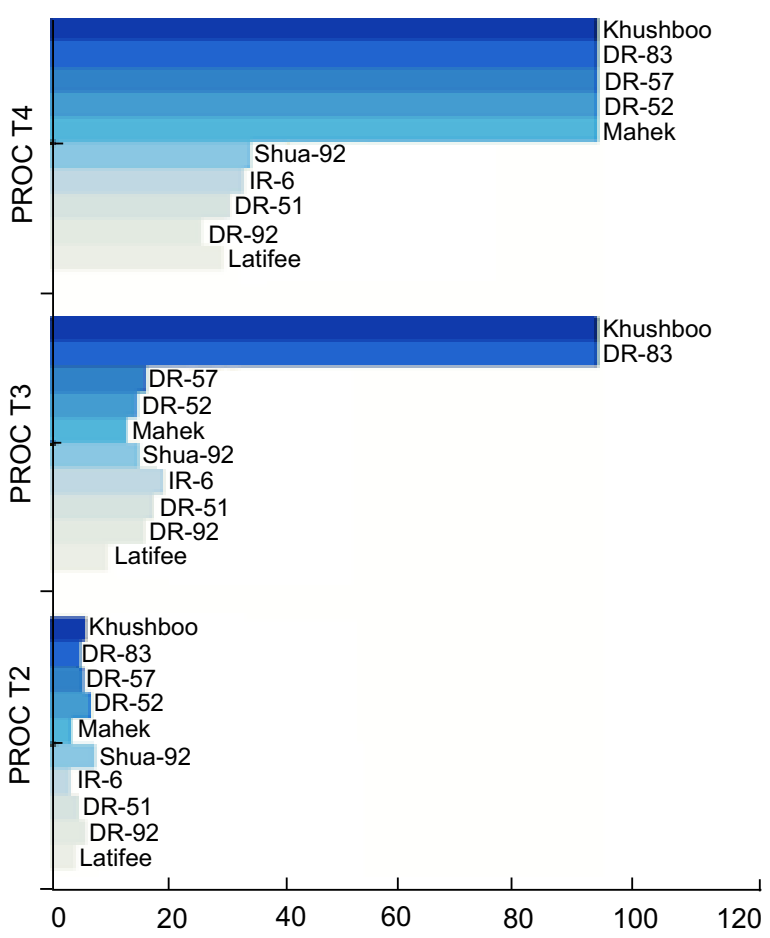

(c) Shoot height percentage increased with the increasing intensity of salinity. This may have resulted from the imbalance of osmotic pressure caused by the concentration of salts (Anbumalarmathi and Mehta, 2013). Other studies

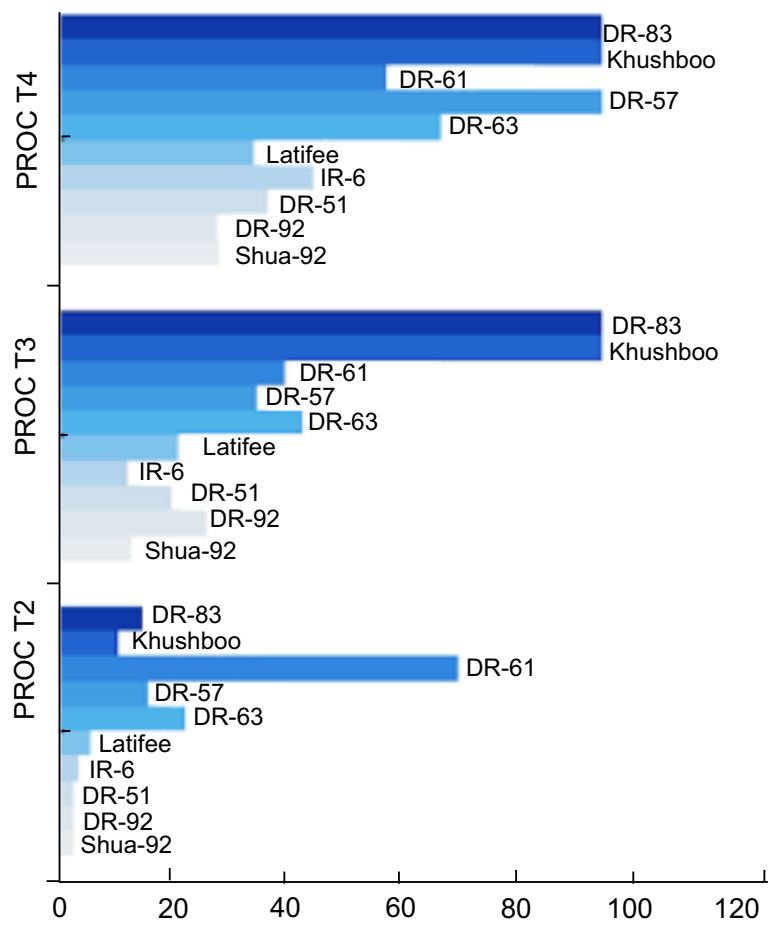

(b) Survival percentage

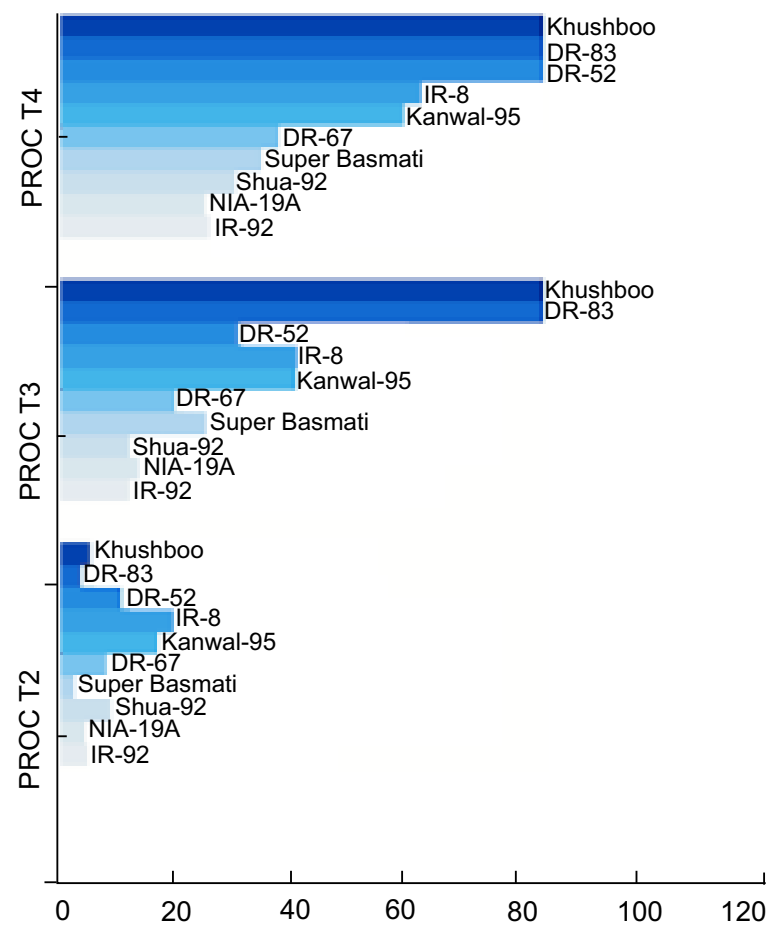

(d) Root length 


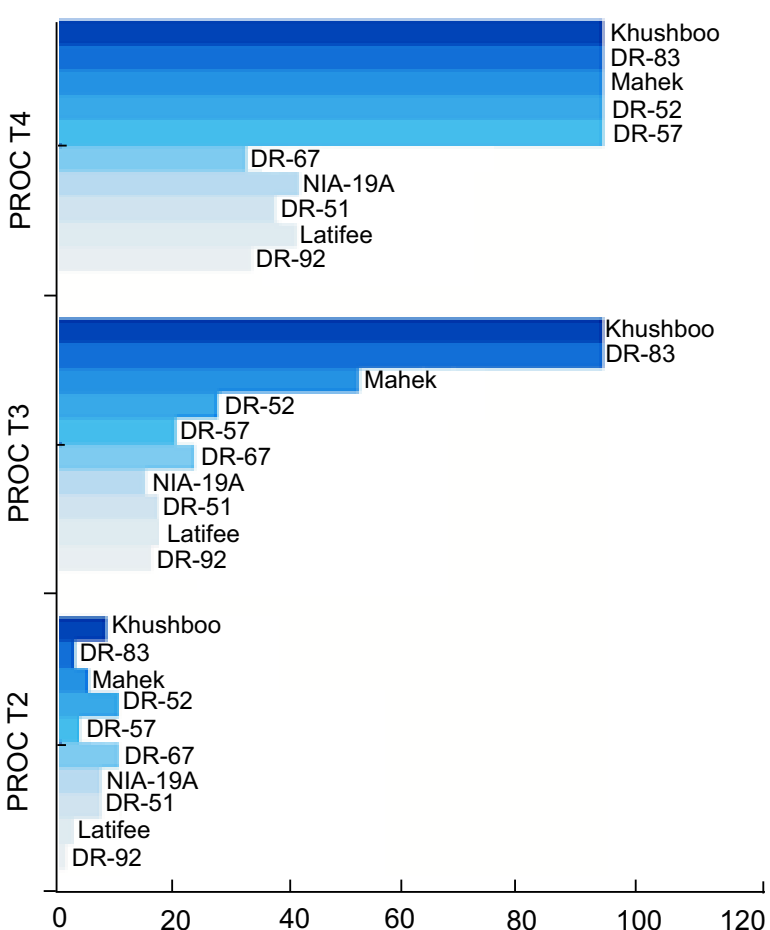

(e) Shoot dry weight

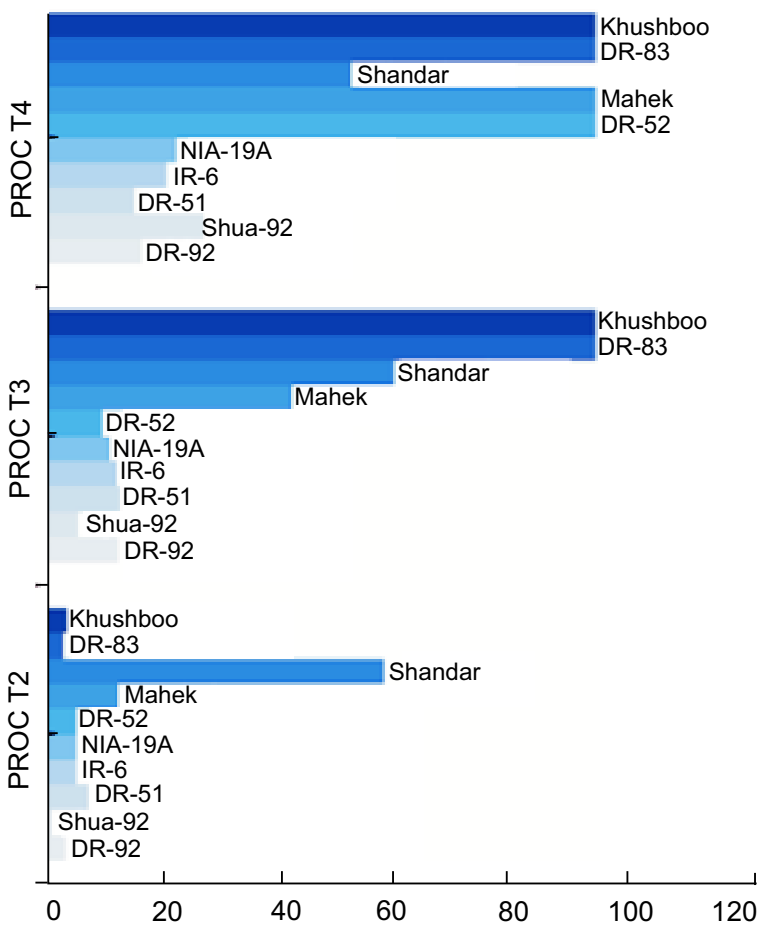

(g) Shoot root ratio
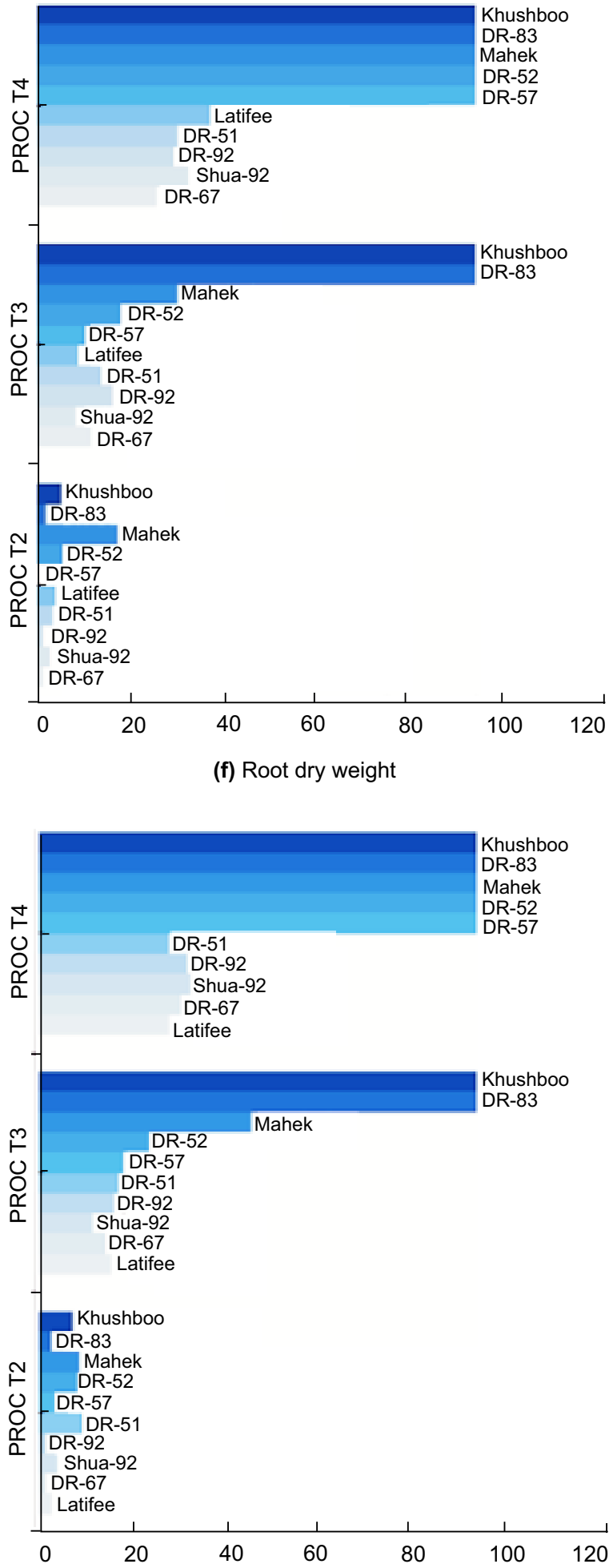

(h) Dry matter production

Fig. 1. Reduction (PROC) in the agro-morphological parameters of rice genotypes at three salinity treatment. (a) Germination percentage, (b) Survival percentage, (c) Shoot height, (d) Root length, (e) Shoot weight, (f) Root dry weight, (g) Shoot root ratio and (h) Dry matter production. Only ten genotypes having the highest and lowest impact of salinity are plotted in the figure for the clarity. 
(Hakim et al., 2010; Zafar et al., 2015) have also reported rice genotypes susceptible for germination percentage against salinity stress particularly in salt sensitive varieties. Germination percentage is among many other parameters that are considered to identify salt-tolerant genotypes. However, a single trait cannot be relied for evaluating salt tolerance because germination depends upon various other factors studied by Ashraf et al. (2006).

Salinity also showed harmful effect on the survival percentage of all aromatic and non-aromatic rice genotypes in present experiment. At all salinity treatments, genotypes DR-92, DR-51 and IR-6 performed better and showed the lowest PROC, whereas the genotypes Khushboo, DR-83, DR-50, DR-52 and Mahek performed meager and showed the highest PROC. Intense reduction in seedling survival and growth is associated with saline environment (Puvanitha and Mahenderan, 2017; Zeng and Shannon, 2000). Roots can be an important trait for the identification of salt tolerant genotypes as these are directly affected by salt concentration (Khan et al., 2007).

In this study the root length decreased in all genotypes when exposed to higher salinity levels. The maximum PROC regarding root length was observed in genotypes

Table 4. Effect of salinity on root length and root dry weight of aromatic and non-aromatic rice genotypes grown in solution culture $\left(\mathrm{NaCl}+\mathrm{CaCl}_{2} @ 20: 1\right.$ salt concentrations $(\mathrm{mM})$

\begin{tabular}{|c|c|c|c|c|c|c|c|c|c|c|}
\hline \multirow[t]{2}{*}{ Genotypes } & \multicolumn{5}{|c|}{ Root length $(\mathrm{cm})$} & \multicolumn{5}{|c|}{ Root dry weight (g) } \\
\hline & $\mathrm{T} 1$ & $\mathrm{~T} 2$ & T3 & $\mathrm{T} 4$ & $\begin{array}{l}\text { Genotype } \\
\text { mean }\end{array}$ & $\mathrm{T} 1$ & $\mathrm{~T} 2$ & T3 & $\mathrm{T} 4$ & $\begin{array}{l}\text { Genotype } \\
\text { mean }\end{array}$ \\
\hline Sarshar & 3.7 & 3.3 & 2.8 & 1.1 & 2.7 & 0.043 & 0.037 & 0.033 & 0.023 & 0.034 \\
\hline Shadab & 3.7 & 3.4 & 2.9 & 1.3 & 2.8 & 0.035 & 0.032 & 0.028 & 0.023 & 0.030 \\
\hline Shandar & 3.3 & 3.0 & 2.5 & 1.5 & 2.6 & 0.025 & 0.023 & 0.020 & 0.012 & 0.020 \\
\hline Shua-92 & 3.5 & 3.1 & 3.0 & 2.2 & 2.9 & 0.054 & 0.052 & 0.049 & 0.035 & 0.048 \\
\hline NIA-19A & 3.3 & 3.1 & 2.7 & 2.3 & 2.8 & 0.048 & 0.043 & 0.039 & 0.035 & 0.041 \\
\hline NIA-625 & 3.6 & 3.3 & 2.6 & 1.5 & 2.7 & 0.043 & 0.038 & 0.033 & 0.028 & 0.036 \\
\hline DR-50 & 2.8 & 2.5 & 2.1 & 1.2 & 2.2 & 0.039 & 0.033 & 0.028 & 0.017 & 0.029 \\
\hline DR-57 & 3.7 & 3.1 & 2.8 & 0.0 & 2.4 & 0.034 & 0.034 & 0.030 & 0.000 & 0.025 \\
\hline DR-83 & 3.3 & 3.1 & 0.0 & 0.0 & 1.6 & 0.040 & 0.039 & 0.000 & 0.000 & 0.020 \\
\hline DR-52 & 3.7 & 3.2 & 2.3 & 0.0 & 2.3 & 0.046 & 0.043 & 0.037 & 0.000 & 0.032 \\
\hline DR-51 & 3.9 & 3.5 & 2.7 & 2.3 & 3.1 & 0.046 & 0.044 & 0.039 & 0.031 & 0.040 \\
\hline DR-82 & 3.8 & 3.7 & 2.3 & 1.8 & 2.9 & 0.037 & 0.032 & 0.029 & 0.022 & 0.030 \\
\hline DR-92 & 4.1 & 3.8 & 3.5 & 2.8 & 3.5 & 0.051 & 0.050 & 0.042 & 0.035 & 0.045 \\
\hline IR-6 & 3.8 & 3.7 & 2.3 & 2.0 & 2.9 & 0.045 & 0.043 & 0.037 & 0.028 & 0.038 \\
\hline IR-8 & 5.0 & 3.8 & 2.5 & 1.2 & 3.1 & 0.047 & 0.040 & 0.039 & 0.025 & 0.038 \\
\hline Sada Hayat & 3.9 & 3.5 & 2.6 & 1.6 & 2.9 & 0.034 & 0.030 & 0.027 & 0.021 & 0.028 \\
\hline Kanwal-95 & 5.0 & 4.0 & 2.6 & 1.4 & 3.2 & 0.040 & 0.038 & 0.036 & 0.021 & 0.034 \\
\hline Shahkar & 4.0 & 3.6 & 2.5 & 1.8 & 3.0 & 0.047 & 0.044 & 0.039 & 0.032 & 0.041 \\
\hline DR-59 & 4.0 & 3.9 & 2.8 & 1.5 & 3.1 & 0.034 & 0.031 & 0.027 & 0.022 & 0.029 \\
\hline Mahek & 2.6 & 2.4 & 2.1 & 0.0 & 1.8 & 0.037 & 0.030 & 0.025 & 0.000 & 0.023 \\
\hline Khushboo & 2.8 & 2.6 & 0.0 & 0.0 & 1.3 & 0.033 & 0.031 & 0.000 & 0.000 & 0.016 \\
\hline DR-62 & 3.2 & 2.5 & 2.0 & 1.0 & 2.2 & 0.041 & 0.040 & 0.037 & 0.017 & 0.034 \\
\hline DR-66 & 3.9 & 3.3 & 2.8 & 1.9 & 3.0 & 0.045 & 0.043 & 0.039 & 0.024 & 0.038 \\
\hline DR-67 & 3.1 & 2.8 & 2.4 & 1.7 & 2.5 & 0.047 & 0.046 & 0.041 & 0.034 & 0.042 \\
\hline DR-63 & 2.7 & 2.2 & 2.2 & 1.2 & 2.1 & 0.046 & 0.040 & 0.036 & 0.024 & 0.037 \\
\hline Super Basmati & 3.2 & 3.1 & 2.2 & 1.9 & 2.6 & 0.048 & 0.042 & 0.035 & 0.027 & 0.038 \\
\hline DR-61 & 3.7 & 3.0 & 2.5 & 1.4 & 2.6 & 0.035 & 0.030 & 0.025 & 0.018 & 0.027 \\
\hline Latifee & 5.1 & 4.4 & 3.5 & 2.9 & 4.0 & 0.040 & 0.038 & 0.036 & 0.024 & 0.035 \\
\hline \multirow[t]{2}{*}{ Treatment Mean } & 3.66 & 3.24 & 2.39 & 1.41 & & 0.041 & 0.038 & 0.032 & 0.021 & \\
\hline & & \multicolumn{2}{|c|}{ Genotype (G) } & Salinity & GX S & & \multicolumn{2}{|c|}{ Genotype (G) } & Salinity & GX S \\
\hline \multicolumn{2}{|l|}{ S.E.D } & \multicolumn{2}{|l|}{0.11} & 0.04 & 0.22 & & \multicolumn{2}{|l|}{1.47} & 5.58 & 2.95 \\
\hline \multicolumn{2}{|c|}{ L.S.D $(0.05 \%)$} & \multicolumn{2}{|c|}{$0.22 * * *$} & $0.08 * * *$ & $0.44 * * *$ & & \multicolumn{2}{|c|}{$2.91 * * *$} & $11.17 * * *$ & $5.82 * * *$ \\
\hline
\end{tabular}


Khushboo, DR-83 and DR-52, whereas minimum PROC was recorded in genotypes DR-92, NIA-19A and Super Basmati. Puvanitha and Mahenderan (2017) has also reported the decrease in root length under saline environment in rice. The reduction in root length with increase in salt-stress might be due to the inhibitory effect of sodium chloride salt (Rahman et al., 2001). Similarly, the significant effect of salinity regarding shoot height was observed in all genotypes. A reduction of seedling is a general phenomenon of various crops in saline environment (Hakim et al., 2010).
Puvanitha and Mahenderan (2017) also proved the hazardous influence of salinity on shoot height in rice crop especially in salt susceptible varieties. Results from the present experiments showed reduction in shoot and root dry weights of rice significantly with increased salinity treatments. The highest reduction in shoot and root was observed at $120 \mathrm{mM}$ salinity, whereas the lowest reduction was recorded at $40 \mathrm{mM}$ salinity as compared to control treatment. Hakim et al. (2014) reported that the reduction in shoot and root dry weight due to decreased per unit photosynthesis leaf area. This

Table 5. Effect of salinity on root shoot ratio and total dry matter production of aromatic and non-aromatic rice genotypes grown in solution culture. The rice genotypes against salinity tolerance on the basis of total dry matter production (g/10 plants)

\begin{tabular}{|c|c|c|c|c|c|c|c|c|c|c|c|c|}
\hline \multirow[t]{2}{*}{ Genotypes } & \multicolumn{5}{|c|}{ Root shoot ratio } & \multicolumn{4}{|c|}{ Total dry matter production } & \multicolumn{3}{|c|}{$\begin{array}{l}\text { Tolerance at salinity } \\
\text { level }\end{array}$} \\
\hline & $\mathrm{T} 1$ & $\mathrm{~T} 2$ & $\mathrm{~T} 3$ & $\mathrm{~T} 4$ & $\begin{array}{l}\text { Genotype } \\
\text { mean }\end{array}$ & $\mathrm{T} 1$ & $\mathrm{~T} 2$ & $\mathrm{~T} 3$ & $\mathrm{~T} 4$ & $\mathrm{~T} 2$ & $\mathrm{~T} 2$ & T3 \\
\hline Sarshar & 2.10 & 2.03 & 1.69 & 1.00 & 1.70 & 0.121 & 0.115 & 0.105 & 0.054 & $\mathrm{~T}$ & $\mathrm{~T}$ & MS \\
\hline Shadab & 2.77 & 2.40 & 2.16 & 0.91 & 2.06 & 0.133 & 0.117 & 0.100 & 0.049 & $\mathrm{~T}$ & MT & $\mathrm{S}$ \\
\hline Shandar & 3.53 & 1.35 & 1.29 & 1.56 & 1.93 & 0.132 & 0.113 & 0.088 & 0.055 & $\mathrm{~T}$ & MT & MS \\
\hline Shua-92 & 2.41 & 2.38 & 2.26 & 1.72 & 2.19 & 0.185 & 0.176 & 0.161 & 0.12 & $\mathrm{~T}$ & $\mathrm{~T}$ & MT \\
\hline NIA-19A & 1.96 & 1.85 & 1.73 & 1.49 & 1.76 & 0.14 & 0.125 & 0.112 & 0.084 & $\mathrm{~T}$ & $\mathrm{~T}$ & MT \\
\hline NIA-625 & 1.94 & 1.87 & 1.80 & 0.84 & 1.61 & 0.128 & 0.114 & 0.093 & 0.082 & $\mathrm{~T}$ & MT & MT \\
\hline DR-50 & 2.16 & 2.02 & 1.65 & 1.55 & 1.84 & 0.115 & 0.106 & 0.086 & 0.046 & $\mathrm{~T}$ & MT & MS \\
\hline DR-57 & 2.34 & 2.25 & 2.14 & 0.00 & 1.68 & 0.116 & 0.111 & 0.093 & 0.000 & $\mathrm{~T}$ & $\mathrm{~T}$ & $\mathrm{~S}$ \\
\hline DR-83 & 1.91 & 1.84 & 0.00 & 0.00 & 0.94 & 0.114 & 0.110 & 0.000 & 0.000 & $\mathrm{~T}$ & $\mathrm{~S}$ & $\mathrm{~S}$ \\
\hline DR-52 & 1.80 & 1.70 & 1.62 & 0.00 & 1.28 & 0.129 & 0.117 & 0.096 & 0.000 & $\mathrm{~T}$ & MT & $\mathrm{S}$ \\
\hline DR-51 & 2.70 & 2.49 & 2.33 & 2.26 & 2.44 & 0.172 & 0.154 & 0.140 & 0.120 & $\mathrm{~T}$ & $\mathrm{~T}$ & MT \\
\hline DR-82 & 2.22 & 2.02 & 1.85 & 1.62 & 1.93 & 0.118 & 0.105 & 0.090 & 0.060 & $\mathrm{~T}$ & MT & MS \\
\hline DR-92 & 2.75 & 2.65 & 2.38 & 2.27 & 2.51 & 0.175 & 0.171 & 0.144 & 0.115 & $\mathrm{~T}$ & $\mathrm{~T}$ & MT \\
\hline IR-6 & 2.58 & 2.43 & 2.24 & 2.01 & 2.31 & 0.162 & 0.150 & 0.118 & 0.109 & $\mathrm{~T}$ & MT & MT \\
\hline IR-8 & 1.65 & 1.54 & 1.15 & 0.73 & 1.27 & 0.125 & 0.114 & 0.091 & 0.056 & $\mathrm{~T}$ & MT & MS \\
\hline Sada Hayat & 2.40 & 2.22 & 2.08 & 1.59 & 2.07 & 0.116 & 0.111 & 0.087 & 0.067 & $\mathrm{~T}$ & MT & MS \\
\hline Kanwal-95 & 2.21 & 1.95 & 1.89 & 1.68 & 1.93 & 0.118 & 0.105 & 0.097 & 0.060 & $\mathrm{~T}$ & $\mathrm{~T}$ & MS \\
\hline Shahkar & 2.50 & 2.44 & 1.95 & 1.88 & 2.19 & 0.164 & 0.154 & 0.127 & 0.095 & $\mathrm{~T}$ & MT & MS \\
\hline DR-59 & 2.22 & 2.02 & 1.85 & 1.58 & 1.92 & 0.11 & 0.101 & 0.087 & 0.055 & $\mathrm{~T}$ & $\mathrm{~T}$ & MS \\
\hline Mahek & 3.37 & 2.92 & 1.86 & 0.00 & 2.04 & 0.143 & 0.129 & 0.073 & 0.000 & $\mathrm{~T}$ & MS & $\mathrm{S}$ \\
\hline Khushboo & 3.08 & 2.96 & 0.00 & 0.00 & 1.51 & 0.134 & 0.123 & 0.000 & 0.000 & $\mathrm{~T}$ & $\mathrm{~S}$ & $\mathrm{~S}$ \\
\hline DR-62 & 2.10 & 1.93 & 1.68 & 1.50 & 1.80 & 0.125 & 0.112 & 0.082 & 0.047 & $\mathrm{~T}$ & MT & S \\
\hline DR-66 & 2.41 & 1.76 & 1.66 & 1.23 & 1.76 & 0.153 & 0.137 & 0.116 & 0.088 & $\mathrm{~T}$ & MT & MS \\
\hline DR-67 & 2.22 & 2.10 & 1.90 & 1.70 & 1.98 & 0.128 & 0.125 & 0.108 & 0.086 & $\mathrm{~T}$ & $\mathrm{~T}$ & MT \\
\hline DR-63 & 1.71 & 1.64 & 1.59 & 1.06 & 1.50 & 0.126 & 0.120 & 0.102 & 0.049 & $\mathrm{~T}$ & $\mathrm{~T}$ & $\mathrm{~S}$ \\
\hline Super Basmati & 1.74 & 1.65 & 1.50 & 1.30 & 1.55 & 0.146 & 0.130 & 0.113 & 0.073 & $\mathrm{~T}$ & MT & MS \\
\hline DR-61 & 1.74 & 1.68 & 1.53 & 1.15 & 1.52 & 0.117 & 0.113 & 0.091 & 0.045 & $\mathrm{~T}$ & MT & $\mathrm{S}$ \\
\hline Latifee & 2.70 & 2.66 & 2.45 & 1.86 & 2.42 & 0.146 & 0.141 & 0.121 & 0.102 & $\mathrm{~T}$ & $\mathrm{~T}$ & MT \\
\hline \multirow{2}{*}{\multicolumn{2}{|c|}{ Treatment mean 2.33}} & 2.10 & 1.72 & 1.23 & & & & & & & & \\
\hline & & \multicolumn{3}{|c|}{ Genotype (G) } & Salinity & & \multicolumn{3}{|l|}{ GX S } & & & \\
\hline \multicolumn{2}{|l|}{ S.E.D } & \multicolumn{3}{|c|}{0.07 (Genotype) } & $0.02(\mathrm{Sal}$ & & \multicolumn{3}{|c|}{0.15 (GXS) } & & & \\
\hline \multicolumn{2}{|c|}{ L.S.D $(0.05 \%)$} & \multicolumn{3}{|c|}{$0.15 * * *$} & $0.05 *$ & & \multicolumn{3}{|c|}{$0.30 * * *$} & & & \\
\hline
\end{tabular}

Note: $\mathrm{T}=$ Tolerant $; \mathrm{MT}=$ Moderately tolerant; $\mathrm{MS}=$ Moderately sensitive; $\mathrm{S}=$ Sensitive 
result in inadequate supply of starch needed for shoot growth and decreased turgor, resulting in lower water potential and imbalance supply of nutrients in saline environment.

The shoot/root ratio may also be a good criterion for screening of salt-tolerant crop species. In the current study, a significant difference was observed amongst all genotypes under saline environment as compared to control and this ratio was decreased with increased treatment of salinity. Our findings are in consensus with the findings of Pradheeban et al. (2017) that salinity significantly reduces shoot/root ratio due to toxic effects of $\mathrm{NaCl}$ salt.

\section{Conclusion}

The conclusion is that, the higher concentration of salts inhabited the germination of seeds and effects also on growth of seedlings in various rice genotypes. The dry shoots and roots, ratio yields of all aromatic and nonaromatic genotypes were significantly decreased with increased salinity levels. Compared to other genotypes the DR-92, DR-51, IR-6, Latifee and DR-67 genotypes were less affected by salinity at all treatments. While, the Mahek, Khusboo and DR-83 genotypes are ranked as salt-sensitive in nature, further studies in laboratory and field conditions at other growth and development stages are proposed to establish and classify the salt-tolerant and salt-sensitive genotypes of rice.

\section{Acknowledgment}

We are thankfull to the Centre for Bio-Saline Agriculture, Tandojam, Department of Soil Science, Sindh Agriculture University, Tandojam, and Department of Botany, Shah Abdul Latif University, Khairpur for their technical support. We are also grateful to Rice Research Institute Dokri, district Larkana, Sindh, Pakistan and Nuclear Institute of Agriculture Tandojam, Sindh, Pakistan for providing seeds of rice. We are also grateful to the two anonymous reviewers for their valuable comments for the improvement of manuscript.

Conflict of Interest. The authors declare no conflict of interest.

\section{References}

Ahmad, M.S.A., Ashraf, M., Ali, Q. 2010. Soil salinity as a selection pressure is a key determinant for the evolution of salt tolerance in blue panicgrass (Panicum antidotale Retz.). Flora, 205: 37-45.

Ali, M.N., Ghosh, B., Saikat, G., Chakraborty, S. 2014. Selection of rice genotypes for salinity tolerance through morpho-biochemical assessment. Journal of Rice Science, 21: 288-298.

Anbumalarmathi, J., Mehta, P. 2013. Germination of indica rice varieties. European Journal of Biological Sciences, 6: 1-6.

Ashraf, M. 2010. Inducing drought tolerance in plants: some recent advances. Biotechnology Advances, 28: $169-183$.

Ashraf, M.A., Shahid, A.A., Rao, A.Q., Bajwa, K.S., Hussain, T. 2006. Functional characterization of a bi-directional plant promoter from cotton leaf curl Burewala virus using an Agrobacterium mediated transient assy. Viruses, 6: 223-242.

Awan, M., Abass, M., Muzafar, A., Ali, A., Tabasum, B. 2015. Transformation of insect and herbicide resistance gene in cotton (Gosipium hirsutum L.). Journal of Agricultural Science and Technology, 17: 287-298.

Ganie, S.A, Borgohain, M.J., Kritika, K., Talukdar, A., Pani, D.R., Mondal, T.K. 2016. Assessment of genetic diversity of Saltol QTL among the rice (Oryza sativa L.) genotypes. Physiology Molecular Biology of Plants, 22: 107-114.

Ghosh, B., Ali, M.N., Saikat, G. 2016. Response of rice under salinity stress: a review opdate. Journal of Research of Rice, 4: 167. doi: 10.4172/2375-4338. 1000167.

Gopalan C., Rama, S.B.V., Balasubramanian, S. 2007. Nutritive Value of Indian Foods. National Institute of Nutrition (NIN), ICMR (Indian Council of Medical Research).

Hakim, M.A., Juraimi, A.S., Begum, M., Hanafi, M.M., Ismail, M.R., Selamat, A. 2010. Effect of salt stress on germination and early seedling growth of rice (Oryza sativa L.). African Journal of Biotechnology, 9: 1911-1918.

Hakim, M.A., Juraimi, S., Hanafi, M.M., Ismail, M.R., Rafii, Y.M., Islam M.M., Selamat, A. 2014. Effect of salinity on growth, ion accumulation and yield of rice varieties. Journal of Animal and Plant Sciences, 24: 874-885.

Hussain, S.S., Jamil, M., Sadiq, M., Mehdi, S.M. 2012. Alleviation of adverse effect of first irrigation to wheat crop in saline sodic soil. Soil and Environment, 31: 78-82.

Huyen, L.T.N., Cuc, L.M., Ham, L.H., Khanh, T.D. 2013. Introgression the Saltol QTL into Q5BD, the elite variety of Vietnam using marker assisted selection (MAS). American Journal of Bioscience, 1: $80-84$.

Islam, M.R., Salam, M.A., Hassan, L., Collard, B.C., Singh, R.K., Gregorio, G.B. 2013. QTL mapping for salinity tolerance at seedling stage in rice. Emirates 
Journal of Foods and Agriculture, 23: 137-146.

Khan, M., Yasmin, S., Ansari, R., Sherazi, M., Ashraf, M. 2007. Screening for salt tolerance in wheat genotypes at an early seedling stage. Pakistan Journal of Botany, 39: 2501-2509.

Kronzucker, H.J., Szczerba, M.W., Schulze, L.M., Britto., D.T. 2008. Non reciprocal interaction between $\mathrm{K}^{+}$and $\mathrm{Na}^{+}$ions in barly (Hordeum vulgar L.). Journal of Experimental Botany, 59: 2793-2801.

Läuchli, A., Grattan, S.R. 2007. Plant growth and development under salinity stress. In: Advances in Molecular Breeding Toward Drought and Salt Tolerant Crops. M. A. Jenks, P. M. Hasegawa and S. M. Jain (eds.), Springer, The Netherlands.

Meloni, D.A., Oliva, M.A., Ruiz, H.A., Martinez, C.A. 2001. Contribution of proline and inorganic solutes to osmotic adjustment in cotton under salt stress. Journal of Plant Nutrition, 24: 599-612.

Mohammadi-Nejad, G., Singhb, R.K., Arzanic, A., Rezaiec, A.M., Sabourid, H., Gregoriob, G.B. 2010. Evaluation of salinity tolerance in rice genotypes. International Journal of Plant Production, 4: 17356814.

Molla, K.A., Debnath, A.B., Ganie, S.A., Mondal, T.K. 2015. Identification and analysis of novel salt responsive candidate gene based SSRs (cgSSRs) from rice (Oryza sativa L.). BMC Plant Biology, 15: 122. https://doi.org/10.1186/s12870-015-0498-1.

Mondal, S., Borromeo, T.H. 2016. Screening of salinity tolerance of rice at early seedling stage. Journal of Bioscience and Agriculture Research, 10: 843-847.

Munns, R., Tester, M. 2008. Mechanisms of salinity tolerance. Annual Review of Plant Biology, 59: 651-681.

Muzaffar, A., Kiani, S. Khan, M.A. Rao, A.Q., Ali, A. 2015. Chloroplast localization of Cry $1 \mathrm{AC}$ and Cry2A protein-an alternative way of insect control in cotton. Biological Research, 48: 14. DOI 10.1186/ s40659-015-0005-z

Nasir, I.A., Tabasum, B., Qamar, Z., Javedm, M.A., Tariq, M. 2014. Herbicide-tolerant sugar (Saccharum officinarum L.) plants: unconventional method of weed removal. Turkish Journal of Biology, 37: 472-478.

Nazar, R., Iqbal, N., Masood, A., Syeed, S., Khan, N.A. 2011. Understanding the significance of sulfur in improving salinity tolerance in plants. Environmental and Experimental Botany, 70: 80-87.

Noreen, Z., Ashraf, M., Akram, N.A. 2010. Salt-induced modulation in some key gas exchange characteristics and ionic relations in pea (Pisum sativum L.) and their use as selection criteria. Crop and Pasture Science, 61: 369-378.

Ologundudu, A., Adelusi, A.A., Akinwale, R.O. 2014. Effect of salt-stress on germination and growth parameters of rice (Oryza sativa L.). Notulae Scientia Biologicae, 6: 237-43.

PBS, Pakistan Bureau of Statistics, 2018. Agricultural Statistics Division, Pakistan Bureau of Statistics, Government of Pakistan www.pbs.gov.pk

Pradheeban, L., Nissanka, S.P, Suriyagoda, L.D.B. 2017. Influence of whole and sub soil salinity on growth, development, physiology and yield of selected rice varieties cultivated in Jaffna district. Sri Lanka Tropical Agricultural Research, 28: 389-401.

Pushpam, R., Rangasamy, S.S. 2000. Invitro response of rice genotypes to salt stress. Madras Agricultural Journal, 27: 694-697.

Puvanitha, S., Mahenderan, S. 2017. Effect of salinity on plant height, shoot and root dry weight of selected rice cultivars. Scholars Journal of Agriculture and Veterinary Sciences, 4: 126-131.

Rahman, M.S., Miyake, H., Taheoka, Y. 2001. Effect of sodium chloride salinity on seed germination and early seedling growth of rice (Oryza sativa L.). Pakistan Journal of Biological Sciences, 4: 351-355.

Rao, A.Q., Bajwa, K.S., Puspito, A.N., Khan, N., Abbas, M.A. 2013. Variation in expression of phytochrome B gene in cotton (Gosipium hirsutum L.). Journal of Agricultural Science and Technology, 15: 10331042.

Raun, S., Xue, Q., Thlkowska, K. 2002. Effect of seed priming on germination and health of rice (Oryza sativa L.) seeds. Seed Science and Technology, 30: 451-458.

Roy, S.J., Negrao, S., Tester, M. 2014. Salt resistant crop plants. Current Opinion in Biotechnology, 26: 115-124.

Sankar, P.D., Saleh, M.A., Selvaraj, C.I. 2011. Rice breeding for salt tolerance. Research in Biotechnology, 2: 1-10.

Shannon, M.C., Rhoders, J.D., Draper, J.H., Scardaci, S.C., Spyres, M.D. 1998. Assessment of salt tolerance in rice cultivars in response to salinity problems in California. Crop Science, 38: 394- 396.

Zafar, S.A., Shoukat, S., Ahmed, H.G., Khan, A., Ali, M.Z., Atif, R.M. 2015. Assessment of salinity tolerance in rice using seedling based morpho-physiology indices. Advancement in Life Sciences, 2: 142-149.

Zeng, L., Shannonm, M.C. 2000. Effects of salinity on grain yield and yield components of rice at different seeding densities. Agronomy Journal, 92: 418-423. 